\title{
Hipotiroidismo subclínico en mujeres embarazadas
}

\author{
Sergio Guerrero Medina*; Bernardo Reyes Leal**
}

RESUMEN: Para estudiar la posibilidad de una relación entre la función Tiroidea de la mujer embarazada y el producto de la gestación 173 mujeres fueron estudiadas. Como test de su función Tiroidea una dosificación de TSH basal y post TRH fue llevada a cabo; del mismo modo se midió el nivel de TSH del recién nacido al quinto día después del parto. Al finalizar el estudio 93 parejas madre-hijo tenían las correspondientes dosificaciones y pudieron ser analizadas.

49 del grupo total de 173 mujeres presentaron valores de TSH post TRH elevados (superiores a $20 \mu \mathrm{U}$.ml) y fueron clasificadas como teniendo "Hipotiroidismo sub-clínico". 3 Recién nacidos tuvieron TSH superior a $30 \mu$ U.ml y fueron considerados como Hipotiroideos congénitos; 4 recién nacidos tuvieron TSH superior a $12 \mu \mathrm{U}$.ml e inferiores a $29 \mu \mathrm{U}$.ml y fueron clasificados como teniendo valores "sub-normales", los demás 86 recién nacidos tuvieron valores normales.

EI análisis de las 93 parejas madre-hijo mostró 78 mujeres con TSH normal, y 15 con niveles elevados. 1 hipotiroideo congénito y un recién nacido con TSH sub-normal se encontraron en el grupo de las 78 mujeres normales, y 1 hipotiroideo Congénito y 2 sub-normales en el grupo de las 15 mujeres hipotiroideas.

Esto representa una probabilidad de 1 sobre 5 en el grupo de mujeres hipotiroideas contra 1 sobre 39, en el grupo de mujeres normales de tener un hijo con Función Tiroidea anormal. Del mismo modo 1 sobre 15 (6.6 p.100) en la mujer Hipotiroidea, contra 1 sobre 78 (1.2 p.100) en la mujer normal de tener un hijo con Hipotiroidismo congénito.

PALABRAS CLAVES: Embarazo, hipotiroidismo sub-clínico TSH post TRH, TSH neo-natal, hipotiroidismo congénito, función tiroidea anormal.

SUMMARY: In order to study the possibility that decreased Thyroid function in the mother could alter thyroid function in the neo-nate, 173 pregnant females were included in this project. As a test of their Thyroid status, Basal and post-THR (200mcg EV) TSH values were obtained. After delivery, in the fifth day TSH was measured in the new borns.

At the end of the study, 93 "couples" mother and her own children, who have had their TSH measured could be analysed.

49 of the total group of 173 mothers presented high post-TRH-TSH levels (above $20 \mu \mathrm{U}$.ml) and were classified as "Sub-Clinical Hypothyroid".

3 Neo-nates had TSH values above $30 \mu \mathrm{U} . \mathrm{ml}$ and were classified as having "Congenital Hypothyroidism"; 4 neo-nates with TSH levels above $12 \mu \mathrm{U} . \mathrm{ml}$ and below $30 \mu \mathrm{U} . \mathrm{ml}$, were qualified as "sub-Normal", the others (86 newborns) have values below or equal to $12 \mu \mathrm{U}$.ml and were considered as "Normals". Analysis of the 93 "couples" mother-children showed 78 mothers with normals TSH levels and 15 with elevated values. 1 Congenital Hypothyroid and 1 newborn with "sub normal" level were found among the 78 normal mothers; and 1 Congenital Hypothyroid and 2 "sub-normals" found from 15 hypothyroid mothers.

This represents a probability of 1:5 in the group of Hypothyroid mothers and of 1:39 in the normal women to have a newborn with abnormal Thyroid function. In the same way 1 over $15(6.6 \%)$ in the hypothyroidmother, compared with 1 over $78(1,2 \%)$ in the normal mother to have a newborn with Congenital Hypothyroidism. function.

KEY WORDS: Pregnancy, sub-clinical hypothyroidism, TSH post TRH, neo-natal TSH, congenital hypothyroidism, abnormal thyroid

\section{Introducción}

La influencia ejercida por las hormonas tiroideas sobre el desarrollo cerebral y neurológico normal en el hombre es ampliamente reconocida en la literatura médica.

Clásicamente se ha aceptado que los ejes Hipofisiario-Tiroideos materno y fetal son independientes ya que la placenta en términos prácticos no permitiría la transferencia de hormonas tiroideas (H.T.) ni de tirotrofina (TSH) desde la madre hacia el feto; así el estado de función tiroidea materna (F.T.M.) no influiría en forma sensible el grado de función tiroidea fetal (F.T.F.) (1-8). Sin embargo, desde 1959 Carr

* Residente IV Endocrinología.

** Profesor Titular y Emérito. Sección Endocrinología. Hospital San Juan de Dios.
E. et al. (9) habían sugerido la transferencia en el ser humano, de H.T. en cantidades adecuadas desde la madre hacia el feto. Actualmente ya existe un buen número de publicaciones que apoyan este punto de vista y en las cuales se demuestra dicha transferencia tanto experimentalmente en ratas (1016), perros (9), y ovejas (17-19), como en humanos desde etapas tempranas del embarazo antes del inicio de la F.T.F.

En el feto humano, a pesar de que la capacidad para sintetizar hormona liberadora de tirotrofina (TRH), TSH y H.T. existe desde el final del primer trimestre, es sólo a partir de la vigésima semana de embarazo cuando la glándula tiroides comienza a secretar cantidades importantes y progresivamente crecientes de Tiroxina (T4), determinando así el inicio de la F.T.F., la cual coincide con el pico de secreción y elevación abrupta de los niveles séricos de TSH y T4 fetales $(2,3,6,7,20)$. 
Man E. y Serunian S. en 1976 demostraron que los hijos de madres hipotiroxinémicas inadecuadamente tratadas tenían un coeficiente intelectual inferior al de aquellos de madres eutiroideas o hipotiroxinémicas adecuadamente tratadas (21).

Así, teniendo en cuenta los siguientes hechos:

A. Que los procesos y condiciones que conducen al crecimiento y maduración cerebral fetal normal, que se inician antes del establecimiento de la F.T.F., dependen de la transferencia de hormona tiroidea materna al feto $(10,11,14-16)$.

B. que se han encontrado tanto T3 (producida por la deyodación local cerebral de T4 $(20,22)$ y T4, como receptores nucleares para triyodotironina (T3) en cerebro de feto a la décima semana, y solamente T4 en otros tejidos a la séptima semana de gestación, mucho antes del inicio de la F.T.F. (23-24).

C. Que el origen de estas hormonas, al menos durante la primera mitad de la vida intrauterina, es exclusivamente materno $(10,11,16,24)$.

Podemos comprender entonces que es de vital importancia asegurar un estado funcional tiroideo adecuado en la mujer embarazada con hipotiroidismo con base en administración de T4 (16), para prevenir las consecuencias deletéreas que la hipotiroxinemia tanto materna como fetal (características de las áreas de bocio endémico) puedan tener sobre el crecimiento y maduración del sistema nervioso y de otros sistemas en el feto que conllevan a alteraciones del desarrollo tanto físico como intelectual de la descendencia $(10,11,16)$.

En estas áreas de bocio endémico, como Colombia, la baja ingesta de yodo en la dieta conduce a hipotiroxinemia materna que se asocia con el nacimiento de hijos sordomudos, con pobre coordinación motora e imbéciles, conocidos como cretinos $(10,11)$, cuyas madres con diferentes grados de hipotiroidismo, tienen niveles subóptimos de H.T. (3), principalmente de T4 (1) (aunque los niveles de T3 puedan permanecer normales o ligeramente elevados (26), probablemente por ser una hormona más potente y de menor costo metabólico) que son evidenciados por una elevación anormal de la TSH tanto basal $(10,11,25,26,27,34)$ como después del estímulo con TRH (25). Estos bajos niveles de T4, además de ser insuficientes para la madre, lo son también para los requerimientos metabólicos y de crecimiento y desarrollo, principalmente neurológico del feto tanto antes del inicio de la F.T.F. (20a. semana) como en la segunda mitad del embarazo. Este déficit de $\mathrm{T} 4$ en el recién nacido prevalecerá aun en la etapa postnatal cuando todavía continúa el crecimiento y desarrollo cerebral $(10,11,16,27)$.

Consideramos que cualquier trabajo que se realice en Colombia y que concierna a las consecuencias deletéreas que para un individuo y un grupo social trae la endemia de bocio se justifica enteramente. Somos concientes de que todos los habitantes de una zona de bocio endémico, tengan o no bocio, sufren consecuencias físicas e intelectuales que contribuyen, probablemente con la desnutrición al retraso cultural y técnico de estos países.

La posibilidad, como ya se mencionó, de que la hipotiroxinemia materna pueda tener como consecuencia un bajo nivel intelectual de su hijo, es una noción de máximo interés (27), y en efecto es muy fácil y muy barato establecer una suplencia tiroidea adecuada en la madre desde el comienzo del embarazo, lo cual permitiría obtener una descendencia con un desarrollo físico e intelectual más adecuado, y prevenir el retardo mental por esta causa $(10,11,21)$.

Basados en estos conceptos y sabiendo que en Colombia, país con endemia de bocio, nacen 20 hipotiroideos congénitos por cada mil nacimientos (35), mientras que en países que no son áreas de bocio endémico nace un cretino por cada 4.000-5.000 nacidos vivos $(1,2,4-7,31)$; quisimos estudiar, como primer objetivo, la incidencia de hipotiroidismo en un grupo escogido al azar, de mujeres embarazadas que consultaron en el Hospital Materno Infatil de Bogotá (H.M.I.) y en segundo lugar, la relación que pudiera existir entre la función tiroidea de la madre y la de su hijo.

\section{Sujetos y métodos}

Entre febrero de 1988 y septiembre de 1989, se tomaron al azar, por método aleatorio simple, de la consulta prenatal del H.M.I., 202 mujeres embarazadas en cualquier etapa de gestación. Tuvimos en cuenta los siguientes criterios de exclusión: se rechazaron todas aquellas que hubieran recibido durante su embarazo o en las últimas seis semanas drogas capaces de alterar la función de la glándula tiroides, como suplencia tiroidea, drogas antitiroideas o que pudieran contener yodo, litio, salicilatos, difenilhidantoinas, estrógenos o progestágenos, B-bloqueadores, glucocorticoides, medios de contraste yodados $(1,2,6)$ o que hubieran sido sometidas a tratamientos médicos, quirúrgicos o radioterápicos susceptibles de modificar la función tiroidea; para efectos de inclusión no se tuvieron en cuenta otras variables clínicas como alteraciones del tamaño tiroideo, procedencia, cifras de tensión arterial, peso, talla, antecedentes familiares o personales de otras alteraciones clínicas, metabólicas, cifras de glicemia, ni de otros datos de laboratorio.

Con base en estas 202 mujeres se hizo la descripción clínica de la población estudiada, y a todas se les tomó muestra de sangre para determinación de sus niveles séricos de TSH tanto basales como 30 minutos después de inyección intravenosa de TRH (TSH-b y post-TRH), por considerarlo el método paraclínico más sensible y específico para el diagnóstico de hipotiroidismo y cuyo comportamiento en el embarazo normal es similar al de mujeres control no embarazadas $(3,4,5,7,29)$; sin embargo, 29 de ellas no figuran en el análisis de los datos de laboratorio por las siguientes razones:

A. Débido a inconvenientes en el almacenamiento y congelación de las muestras hay 25 casos cuyos resultados de laboratorio por ser poco confiables no pueden ser tenidos en cuenta. (Diferencia de más de 10 p.100, entre los duplicados).

B. En 4 casos no se dispone de estos resultados porque las muestras no habían sido procesadas al momento del cierre de la recolección de la información.

Quedan entonces 173 mujeres con datos confiables de laboratorio. Entre las 202 mujeres incluidas, hay un grupo de 73 en las que no fue posible tener conocimiento sobre el desenlace del embarazo ni muestra de sangre de su hijo para determinación de la función tiroidea; para efectos de este trabajo, lo llamaremos "grupo desertor". Entre las 129 mujeres restantes con las que sí obtuvimos esta información, hay un grupo de siete, cuyo embarazo terminó como aborto 
espontáneo, mola hidatiforme, mortinato, o el recién nacido murió antes del quinto día de edad, por lo cual no hay determinación del TSH neonatal (TSH-NN), lo denominaremos "grupo de embarazos anormales". Tenemos así un total de 122 neonatos vivos con datos de laboratorio. Hay una mujer cuyo embarazo fue gemelar y dio nacimiento a 2 bebés vivos que fueron incluidos, arrojando así un total de 23 productos de la gestación de los cuales tuvimos información. En resumen, se conformaron los siguientes grupos de madres y de hijos:

\section{GRUPOS MATERNOS:}

A. Grupo de madres con TSH-b y post-TRH confiable

B. Grupo de madres con TSH-b y post-TRH no confiable

C. Grupo de madres sin TSH-b ni post-TRH

Total

Para efectos de este trabajo, denominaremos entonces a las 73 mujeres del Grupo A, "Grupo Materno de Estudio" (G.M.E.) y a las 29 mujeres que suman los grupos B. y C., "Grupo de mujeres sin TSH". Además, teniendo en cuenta que a la mujer que tuvo el embarazo gemelar, hubo necesidad de contarla dos veces, por ser madre de 2 hijos en estudio y por pertenecer al grupo de mujeres sin TSH, este último grupo queda conformado por 30 mujeres, sumando un total de 203 madres que denominaremos "Grupo Materno Totar' (G.M.T.)

\section{GRUPOS DE HIJOS:}

1. Grupo de hijos con determinación de TSH-NN

2. "Grupo desertor"

3. "Grupo de embarazos anormales"

Total

Al cruzar estos grupos de madres con los grupos de hijos con el fin de hacer las descripciones y correlaciones entre parejas madre-hijo se conforman las parejas que se muestran en el cuadro 1.

Cuadro 1

DISTRIBUCION DE LOS GRUPOS DE PAREJAS

\begin{tabular}{|c|c|c|c|c|}
\hline $\begin{array}{l}\text { Hijos } \\
\text { Madres }\end{array}$ & $\begin{array}{l}\text { Con } \\
\text { TSH-NN }\end{array}$ & $\begin{array}{l}\text { "Deser- } \\
\text { tores" }\end{array}$ & $\begin{array}{l}\text { "Embar. } \\
\text { Anor- } \\
\text { males" }\end{array}$ & Total \\
\hline TSH confiab. & 93 & 73 & 7 & 173 \\
\hline TSH No Conf. & $26^{*}$ & 0 & 0 & $26^{*}$ \\
\hline Sin TSH & 4 & 0 & 0 & 4 \\
\hline Total & $123 *$ & 73 & 7 & 203* \\
\hline
\end{tabular}

* Incluye el embarazo gemelar como doble y 2 hijos nacidos vivos.

Además de las muestras para las determinaciones de TSHb y post-TRH, tomadas a las mujeres al momento de ser incluidas, se obtuvo información respecto de su edad, lugar de nacimiento y procedencia, historia obstétrica previa, edad del embarazo por amenorrea, y determinación por palpación del tamaño de la glándula tiroides. Posteriormente, y siempre que fue posible, se mantuvo contacto telefónico con ellas con el fin de conocer cuándo y cómo terminaba su gestación, e insistiéndoles en la necesidad de llevar a su hijo recién nacido después del quinto día de edad a la sección de endocrinología, para la determinación de la TSH-NN y obtener información sobre la fecha y modalidad del parto (VaginalCesárea), peso y talla al nacer, sexo, duración de la gestación y examen físico del recién nacido (R.N.) en busca de posibles signos de transtorno de la función tiroidea.

La muestra de la madre para determinación de la TSH, se hizo, tanto basal (TSH-b), como 30 minutos después del estímulo con 200 ugrs de TRH (TSH post-TRH) y ambas muestras fueron inmediatamente centrífugadas y el suero congelado a $-20^{\circ} \mathrm{C}$ hasta su procesamiento. Su determinación se hizo por Radioinmunoanálisis (RIA) para lo cual se utilizó un kit comercial (DPC).

Para determinar la TSH-NN se tomó muestra de sangre por punción en el talón a todos los hijos después del quinto día de edad (7) cuando fueron traídos al hospital, o se permitió la visita a sus hogares para la obtención de la muestra y de la información ya referida. La muestra se tomó en papel de filtro y se almacenó refrigerada a $-20^{\circ} \mathrm{C}$ hasta su procesamiento, que se hizo por RIA utilizando un kit comercial (DPC).

Se aceptaron como valores normales en las madres para el TSH-b todos los inferiores a $5 \mu \mathrm{U} / \mathrm{ml}$ y para el nivel post-TRH, todos los inferiores a $20 \mu \mathrm{U} / \mathrm{ml}$. Todas las mujeres con valores iguales o superiores a $5 \mu \mathrm{U} / \mathrm{ml}$ para el TSH-b y/o, iguales o superiores a $20 \mu \mathrm{U} / \mathrm{ml}$ para el TSH post-TRH fueron consideradas como hipotiroideas (29). Respecto de los valores de TSH-NN, se consideraron como normales todos los inferiores a $12 \mu \mathrm{U} / \mathrm{ml}$; los que tuvieron valores superiores a $12 \mu \mathrm{U} / \mathrm{ml}$ fueron divididos en 2 grupos: aquellos con TSH-NN igual o superior a $30 \mu \mathrm{U} / \mathrm{ml}$ fueron catalogados como "Hipotiroideos Congénitos", y los que tuvieron valores entre 13 y 29 se clasificaron como teniendo "Función tiroidea Subnormal".

Con el objeto de establecer una relación entre la F.T.M. y la del R.N. y para la presentación de los datos se siguió el siguiente esquema: para cumplir con el primer objetivo propuesto, que era conocer la proporción de hipotiroidismo en el grupo de estudio, se presentaban de manera descriptiva los datos obtenidos en las mujeres e hijos incluidos, conformen o no pareja, y en los que en por lo menos en uno de ellos se conozca el valor de la determinación del nivel sérico de TSH, esto quiere decir que la información de las parejas en las cuales falta el dato en alguno de los dos miembros, se tuvieron en cuenta solamente para la presentación descriptiva de los datos. Para efecto de establecer una relación entre la F.T.M. con la de su hijo, se utilizaron las 93 parejas en las cuales ambos miembros tuvieron datos confiables de la determinación del nivel sérico de la TSH.

Para esto se hicieron dos tipos de análisis estadísticos: el primero, individual, es decir, pareja por pareja, con el fin de establecer el número de parejas en las cuales el diagnóstico coincide y en cuantas no, por lo tanto podrán formarse seis grupos distribuidos como sigue: 
1. Madres normales con hijos normales.

2. Madres normales con hijos sub-normales.

3. Madres normales con hijos hipotiroideos congénitos.

4. Madres hipotiroideas con hijos normales.

5. Madres hipotiroideas con hijos sub-normales.

6. Madres hipotiroideas con hijos hipotiroideos congénitos.

El segundo tipo de análisis se hizo considerando a todas las madres como un solo grupo, buscando un coeficiente de correlación matemáticamente significatıvo entre la totalidad de los valores maternos de TSH post-TRH (ya que la cifra basal se encontró elevada en sólo 3 madres cuyos valores de TSH post-TRH también estuvieron anormalmente elevados) y los valores de TSH-NN, para así cumplir con el segundo objetivo de este trabajo, que era tratar de conocer la relación que pudiera existir entre la F.T.M. y la de su hijo.

Se investigó además si exisía un coeficiente de correlación estadísticamente significativo entre el estado de la F.T.M. definido por el resultado de la determinación de la TSH-b y post-TRH y otras variables como la edad y el tamaño de la tiroides de la madre, la duración de su embarazo, la edad gestacional al momento del ingreso al estudio, historia obstétrica previa, sexo, peso y talla del hijo al nacer; se buscó también correlación estadísticamente significativa entre estado de la función tiroidea del hijo (FTH) y estas mismas variables.

Las variables fueron definidas así:

A. Edad de la Madre: la edad materna fue dividida por grupos de cinco años así: 15-20; 21-25; 26-30; 3-35; 36-40; 41-45; 45 o más.

B. Graduación del tamaño de la tiroides materna:

Grado O - tiroides no palpable, o palpable sin estar au-

Oa: mentando de tamaño y no visible.

Grado $\mathrm{Ob}$ : tiroides palpable, anormalmente aumentando de tamaño, pero aún no visible.

Grado I: tiroides palpable y visible a menos de un $\mathrm{m}$ de distancia.

Grado II: tiroides palpable y visible a más de un $\mathrm{m}$ de distancia.

Grado III: bocio gigante.

C. Duración del embarazo estudiado:

- Pretérmino: de 37 semanas o menos.

- A término: entre 38 y 42 semanas de duración.

- Postérmino: de 43 semanas o más.

D. Peso del hijo al nacer:

- Bajo peso: de $2.500 \mathrm{~g}$ o menos.

- Peso normal: entre $2.501 \mathrm{~g}$ y $3.500 \mathrm{~g}$.

- Sobrepeso: de $3.500 \mathrm{~g}$ o más.

E. Talla del hijo al nacer:

- Baja talla: de $47 \mathrm{~cm}$ o menos.

- Talla normal: entre $48 \mathrm{~cm}$ y $52 \mathrm{~cm}$.

- Macrosómico: de $53 \mathrm{~cm}$ o más.
F. Edad gestacional al momento del ingreso al estudio agrupada por tres (3) trimestres:

- Primer trimestre: desde el inicio del embarazo hasta la 13a. semana de gestación.

- Segundo trimestre: entre la 14a. y la 17a. semana

- Tercer trimestre: desde la 28a. semana hasta el término.

G. Sexo del hijo:

- Masculino.

- Femenino.

Definimos como "Hipotiroidismo Subclínico"(30) la circunstancia patológica presente en aquellas personas sin síntomas ni signos clínicos de hipofunción tiroidea con niveles séricos de H.T. y TSH-b dentro de límites normales, pero con elevación de la TSH post-TRH igual o superior a 20 $\mu \mathrm{U} / \mathrm{ml}$;demostrando así una deficiente capacidad de frenación de la secreción hipofisiaria de TSH por parte de los niveles circulantes de T4, circunstancia que hace innecesaria la determinación de tiroxina.

\section{Resultados}

Descripción general de la población. El promedio de edad de las 203 mujeres del Grupo Materno Total fue de 26.1 años $+/$ - 6.4 s.d., con cifras límites entre 15 y 48 años; al agrupar en quinquenios de edad (cuadro 2), se observa una mayor frecuencia en el grupo de 26 a 30 años $(31 \%)$. Se trata pues de una población joven, en el cual el $59 \%$ tiene entre 21 y 30 años y sólo el $2 \%$ es mayor de cuarenta.

Cuadro 2

DISTRIBUCION SEGUN GRUPOS DE EDAD MATERNA

\begin{tabular}{lll}
\hline Grupos de edad & Número & $\%$ \\
\hline $15-20$ & 43 & 21.2 \\
$21-25$ & 57 & 28.1 \\
$26-30$ & 63 & 31.0 \\
$31-35$ & 23 & 11.3 \\
$36-40$ & 12 & 5.9 \\
$41-45$ & 3 & 1.5 \\
$46-48$ & 2 & 0.5 \\
\hline Total & 203 & 100.0 \\
\hline
\end{tabular}

Promedio de edad

Desviación estándar

26.1 años.

6.4

La información sobre el lugar de nacimiento y procedencia de los Grupos Maternos Total y de Estudio se detalla en el cuadro 3 .

Del departamento de Cundinamarca proceden 123 mujeres, de las cuales 93 provienen del área urbana de Bogotá D.E.

De los departamentos de Cundinamarca (incluyendo Bogotá D.E.), Boyacá, Santander, Tolima y Caldas, son naturales 175 mujeres $(86.2 \%)$, mientras que las 28 restantes, nacieron en otras zonas del país. En las 173 mujeres del G. M. E. vemos que la distribución es similar, ya que 148 $(85.5 \%)$ son naturales de esos departamentos, con una mayor 
Cuadro 3

LUGAR DE NACIMIENTO Y PROCEDENCIA DE LAS MADRES INCLUIDAS EN EL ESTUDIO

\begin{tabular}{|c|c|c|c|c|c|c|c|c|}
\hline \multirow[t]{2}{*}{$\begin{array}{l}\text { Lugar de } \\
\text { Nacimiento }\end{array}$} & \multirow{2}{*}{$\begin{array}{l}\text { Mujeres } \\
\text { Número }\end{array}$} & \multirow{2}{*}{$\begin{array}{l}\text { Incluidas } \\
\%\end{array}$} & \multicolumn{2}{|c|}{$\begin{array}{l}\text { Lugar de } \\
\text { Procedencia }\end{array}$} & \multicolumn{2}{|c|}{$\begin{array}{l}\text { Mujeres TSH } \\
\text { Confiable }\end{array}$} & \multirow{2}{*}{$\begin{array}{l}\text { Mujeres } \\
\text { Hipotiroideas } \\
\text { Número }\end{array}$} & \multirow[t]{2}{*}{$\begin{array}{l}\text { Porcentaje hipotir. } \\
\text { sobre pobla. total }\end{array}$} \\
\hline & & & Número & $\%$ & Número & $\%$ & & \\
\hline Bogotá D.E. & 93 & 45.8 & 195 & 96 & 80 & 46.24 & 20 & 11.56 \\
\hline Cundinamarca & 30 & 14.77 & 6 & 3 & 24 & $13: 87$ & 10 & 5.78 \\
\hline Boyacá & 23 & 11.33 & 1 & 0.5 & 20 & 11.56 & 4 & 2.31 \\
\hline Santander & 12 & 5.91 & - & - & 10 & 5.78 & 2 & 1.15 \\
\hline Tolima & 12 & 5.91 & - & - & 9 & 5.20 & 2 & 1.15 \\
\hline Caldas & 5 & 2.46 & - & - & 5 & 2.89 & 3 & 1.73 \\
\hline Huila & 5 & 2.46 & - & - & 4 & 2.31 & 1 & 0.57 \\
\hline Antioquia & 3 & 1.47 & - & - & 3 & 1.73 & 1 & 0.57 \\
\hline Meta & 3 & 1.47 & - & - & 2 & 1.15 & 1 & 0.57 \\
\hline Casanare & 2 & 0.98 & - & - & 2 & 1.15 & - & - \\
\hline Valle & 3 & 1.47 & - & - & 2 & 1.15 & 1 & 0.57 \\
\hline Cauca & 2 & 0.98 & - & - & 2 & 1.15 & 1 & 0.57 \\
\hline Quindío & 2 & 0.98 & - & - & 2 & 1.15 & - & - \\
\hline Magdalena & 2 & 0.98 & - & - & 2 & 1.15 & - & - \\
\hline N. de Santander & 1 & 0.49 & - & - & 1 & 0.57 & 1 & 0.57 \\
\hline Atlántico & 1 & 0.49 & - & - & 1 & 0.57 & 1 & 0.57 \\
\hline Sucre & 1 & 0.49 & - & - & 1 & 0.57 & 1 & 0.57 \\
\hline Arauca & 1 & 0.49 & 1 & 0.5 & 1 & 0.57 & - & - \\
\hline Nariño & 1 & 0.49 & - & - & 1 & 0.57 & - & - \\
\hline Risaralda & 1 & 0.49 & - & - & 1 & 0.57 & - & - \\
\hline Total & 203 & $100.0 \%$ & 203 & $100.0 \%$ & 173 & $100.0 \%$ & 49 & 28.32 \\
\hline
\end{tabular}

* Por el número tan reducido el porcentaje no es válido.

frecuencia para Bogotá D.E. de $80(46 \%)$, y resto de Cundinamarca con 24 mujeres (14\%).

En 126 madres (62\%) del Grupo Materno Total (G.M.T.), la glándula tiroides no fue palpable, en tanto que en $25(12.3 \%)$ fue palpable y visible (cuadro 4 ).

Cuadro 4

TAMAÑO TIROIDEO - GRUPO TOTAL DE MADRES

\begin{tabular}{lcc}
\hline Tamaño tiroideo & Número & $\%$ \\
\hline Grado O- Oa & 126 & 62.1 \\
Grado Ob & 52 & 25.6 \\
Grado I & 24 & 11.8 \\
Grado II & 1 & 0.5 \\
\hline Total & 203 & 100.0 \\
\hline
\end{tabular}

El promedio de la duración del embarazo en las 123 mujeres en quienes se conoce este dato fue de 39.4 semanas (cuadro 5).

Cuadro 5

DURACION DEL EMBARAZO

\begin{tabular}{|c|c|c|}
\hline Duración del embarazo & Número & $\%$ \\
\hline Pretérmino (hasta 37 sem.) & 11 & 8.9 \\
\hline A término $(38-42$ sem. $)$ & 110 & 89.4 \\
\hline Postérmino (43 sem. o más) & 2 & 1.6 \\
\hline Total & 123 & 100.0 \\
\hline Promedio de Duración (en semanas) & \multicolumn{2}{|c|}{39.4} \\
\hline Desviación estándar & \multicolumn{2}{|c|}{1.63} \\
\hline Valores extremos & \multicolumn{2}{|c|}{ : $\quad 33-43$ sem. } \\
\hline
\end{tabular}

La edad gestacional al momento de ingreso al estudio de las mujeres del G.M.T. se presenta en el cuadro 6; 98 mujeres (48\%) ingresaron en el tercer trimestre del embarazo, mientras que en el primer trimestre lo hicieron solamente $21(10 \%)$.

\section{EDAD GESTACIONAL AL MOMENTO DEL INGRESO}

\begin{tabular}{lcc}
\hline Edad gestacional & Número & $\%$ \\
\hline Primer trimestre (0-13 sem.) & 21 & 10.3 \\
Segundo trimestre (14-27 sem.) & 84 & 41.4 \\
Tercer trimestre (28-término) & 98 & 48.3 \\
\hline Total & 203 & 100.0 \\
\hline Promedio de edad gestacional en semanas al ingreso $:$ & 26.2 \\
Valores extremos & $:$ & $4-39$
\end{tabular}

De los 123 nacidos vivos, catorce $(11.4 \%)$ tuvieron bajo peso y talla al nacer. El peso y la talla fueron normales en $82(66.7 \%)$, y en 101 bebés $(82 \%)$ respectivamente. El promedio del peso fue $3.191 .3 \mathrm{~g}$ con un rango de $1.550 \mathrm{a}$ $4.430 \mathrm{~g}$ el de la talla fue de $49.7 \mathrm{~cm}$, con un rango de 49 a $54 \mathrm{~cm}$ (cuadro 7). Se observó, que los bebés nacidos antes del término, tenían como era de esperarse menor peso y talla que los nacidos a término. La distribución por sexo de los 123 nacidos fue la esperada, con $50.4 \%$ para el sexo femenino.

\section{Función tiroidea materna y neonatal}

Función tiroidea materna. En este estudio se encontró que 49 de las 173 mujeres del G.M.E. son hipotiroideas, 
Cuadro 7

PESO Y TALLA DE LOS RECIEN NACIDOS

\begin{tabular}{lcclcl}
\hline Peso & Número & $\%$ & Talla & Número & $\%$ \\
\hline Bajo peso & 14 & 11.4 & Baja talla & 14 & 11.4 \\
Normal & 82 & 66.7 & Normal & 101 & 82.1 \\
Sobre-peso & 27 & 22 & Macrosómico & 8 & 6.5 \\
\hline Total & 123 & 100.0 & & 123 & 100.0 \\
\hline
\end{tabular}

$\begin{array}{lllll}\text { Promedio } & : & 3.191 .2 \mathrm{~g} & \text { Promedio } & 49.7 \mathrm{~cm} \\ \text { Desviación estándar } & : & 482.7 & \text { Desviación estándar : } & 2.34\end{array}$

lo cual corresponde a un $28.32 \%$ de hipotiroidismo materno. (Cuadro 8).

$\mathrm{Al}$ analizar la frecuencia de hipotiroidismo de las madres según el lugar de nacimiento, las tasas más altas se encuentran en el departamento de Cundinamarca (excluida Bogotá D.E.) y Caldas que presentan conjuntamente 13 casos (44.8\%), entre las 29 consultantes de esas regiones. En las demás secciones del país la tasa global de hipotiroidismo resultó ser de $25 \%$ (cuadro 1). La interpretación de estos datos es limitada por el tamaño reducido de la muestra y por desconocerse el tiempo de permanencia en sus lugares de nacimiento, y en la zona de mayor influencia del hospital.

Dentro del G.M.E., el promedio para las cifras del TSH-b y TSH post-TRH en las 124 mujeres eutiroideas fue de 1.98 $\mu \mathrm{U} / \mathrm{ml},+/-0.82$ s.d., con un rango de 0.5 a 4.6 ; y de 8.85 $\mu \mathrm{U} / \mathrm{ml},+/-4.01$ s.d., con un rango de 3.20 a 19.0 respectivamente. Para las madres hipotiroideas el promedio para la TSH-b fue de $2.94 \mu \mathrm{U} / \mathrm{ml}$, +/- 0.42 s.d., con un rango de 1.1 a 5.8 , y el de la TSH post-TRH fue de $35.4 \mu \mathrm{U} / \mathrm{ml}$, $+/$ - 13.11 s.d., con un rango de 20 a 61 . Es necesario aclarar que el nivel sérico de la TSH post-TRH en 6 mujeres hipotiroideas excedió el valor máximo medido por el kit de RIA de TSH que fue de $60 \mu \mathrm{U} / \mathrm{ml}$. Para efectos de obtener el promedio de las determinaciones de TSH post-TRH en las mujeres hipotiroideas (y para los cálculos en que fue necesario tener en cuenta esta cifra), utilizamos en estas seis mujeres, arbitrariamente el valor de $61 \mu \mathrm{U} / \mathrm{ml}$, presumiendo que posiblemente el nivel sérico real era mucho mayor; razón por la cual el promedio de las determinaciones séricas de la TSH post-TRH en las mujeres hipotiroideas aquí presentado, puede ser menor que el real. (Cuadro 8).

Cuadro 8

RESULTADOS DE TSH-GRUPO MATERNO EN ESTUDIO

\begin{tabular}{lcc|cc}
\hline & \multicolumn{4}{c}{ Madres } \\
\cline { 2 - 5 } $\begin{array}{l}\text { Prueba } \\
\text { paraclínica }\end{array}$ & \multicolumn{2}{c}{ Eutiroideas $(\mathrm{n}=124)$} & \multicolumn{2}{c}{ Hipotiroideas $(\mathrm{n}=49)$} \\
& Promedio & $\begin{array}{c}\text { Desviación } \\
\text { Estándar }\end{array}$ & Promedio & $\begin{array}{c}\text { Desviación } \\
\text { Estándar }\end{array}$ \\
\hline TSH-basal & 1.98 & 0.82 & 2.94 & 0.46 \\
TSH-Post-TRH & 8.85 & 4.01 & 35.38 & 13.11 \\
\hline
\end{tabular}

Función tiroidea neonatal. De los 123 niños que constituyen la población infantil en estudio se encontraron 7 (5.7\%) con resultados de la determinación de la TSH-NN superiores a $12 \mu \mathrm{U} / \mathrm{ml}$; siendo tres $(2.4 \%)$ de ellos hipotiroideos congé- nitos (TSH $30 \mu \mathrm{U} / \mathrm{ml}$ ) y cuatro (3.3\%) en el rango catalogado como Sub-normales (13 a $29 \mu \mathrm{U} / \mathrm{ml}$ ) (Cuadro 9).

\section{Cuadro 9 \\ CLASIFICACION GRUPO DE HIJOS SEGUN FUNCION TIROIDEA}

\begin{tabular}{llcc}
\hline $\begin{array}{l}\text { Grado de función } \\
\text { Tiroidea según TSN-NN Número }\end{array}$ & $\%$ & \\
\hline Hijos normales & 116 & 94.3 & \\
Anormales & $\begin{array}{l}\text { Sub-normales } \\
\text { Hipotiroideos } \\
\text { Congénitos }\end{array}$ & 4 & 3.3 \\
& & 3 & 2.4 \\
\hline
\end{tabular}

Relación entre las funciones tiroideas materna y neonatal

De los tres hijos hipotiroideos congénitos, uno nació de madre hipotiroidea, otro de madre eutiroidea y en el tercero el dato materno no es confiable; de los cuatro neonatos con función tiroidea subnormal, dos son hijos de madre hipotiroidea, uno de madre normal y en el otro caso no se cuenta con la información sobre el estado de la F.T.M. Se pudieron obtener datos de TSH neo-natal en 93 recién nacidos; así se conformaron 93 parejas madre-hijo en las cuales se conocen ambos niveles de TSH. Son éstas parejas la base para tratar de establecer la relación eventual entre función Tiroidea materna y función Tiroidea fetal (Cuadro 10).

Cuadro 10

RELACION ENTRE LAS FUNCIONES TIROIDEAS MADRE-HIJO

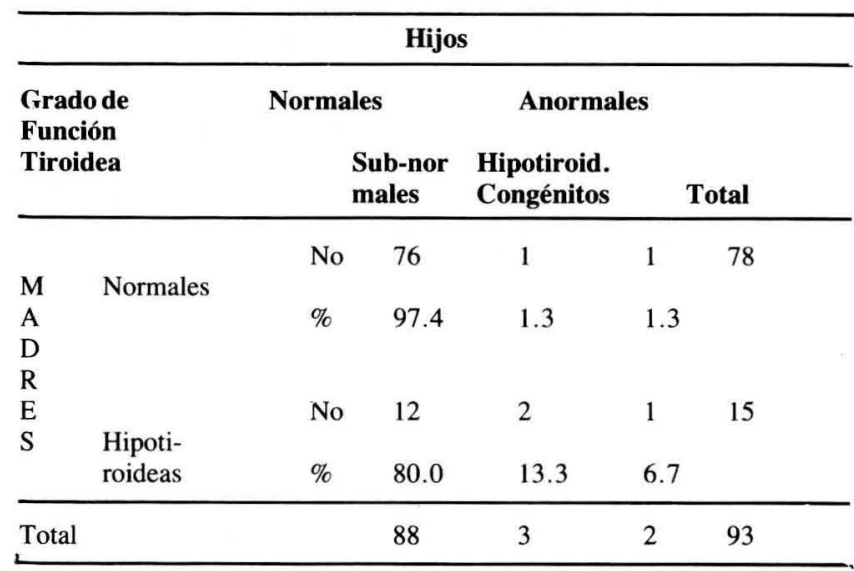

En 79 parejas ( $85 \%$ ) el diagnóstico coincide, de las cuales en $76(97.4 \%)$ madre e hijo son eutiroideos, y en los otros tres casos, madres hipotiroideas dieron nacimiento a hijos anormales ( 2 sub-normales y 1 hipotiroideo congénito). En otros dos casos (2.1\%), de madres eutiroideas nacieron bebés con hipofunción tiroidea (uno sub-normal y otro hipotiroideo congénito) y hubo 12 parejas (13\%) con madres hipotiroideas e hijos normales (eutiroideos). A pesar de que el resultado del cálculo estadístico no muestra una diferencia estadísticamente significativa $(p=0.11)$ entre las dos proporciones, en el cuadro anterior se observan dos fenómenos importantes:

1. Que entre las cinco parejas con hijos con hipofunción tiroidea, hay 3 madres que pertenenecen al grupo de las 15 
hipotiroideas, y solamente dos al grupo de las 78 mujeres normales; mostrando que, en el grupo de población estudiado, el riesgo que una mujer eutiroidea tiene de dar a luz un hijo con hipofunción tiroidea es de 1:39, mientras que en una madre hipotiroidea se aumenta a 1:5. (Ocho veces mayor).

2. Que la posibilidad que tienen los hijos de mujeres hipotiroideas de tener función tiroidea sub-normal es 10 veces mayor que la de los hijos de mujeres eutiroideas, y cinco veces mayor de ser hipotiroideos congénitos.

Estos resultados indican que en nuestro medio este problema amerita seguir siendo estudiado, de manera que, aumentando el tamaño de la muestra a estudiar, permita obtener conclusiones más contundentes y se pueda demostrar una correlación entre la F.T.M. y la neonatal más estrecha, con un nivel de significancia mayor.

\section{Función tiroidea materna y otras variables}

Función y tamaño tiroideo materno. En el cuadro 11 llama la atención que 40 (32\%) de 124 mujeres que siendo eutiroideas, mostraron un aumento del tamaño de la glándula tiroides durante su embarazo, hallazgo ampliamente descrito en la literatura $(3-7,29)$. Esta misma circunstancia ocurrió en 22 de las 49 mujeres hipotiroideas, aunque en una proporción mayor $(45 \%)(1,6,32,33)$. En la población estudiada no se encontró ningún caso con bocio gigante, (grado III).

$$
\text { Cuadro } 11
$$

FUNCION Y TAMAÑO TIROIDEO MATERNO

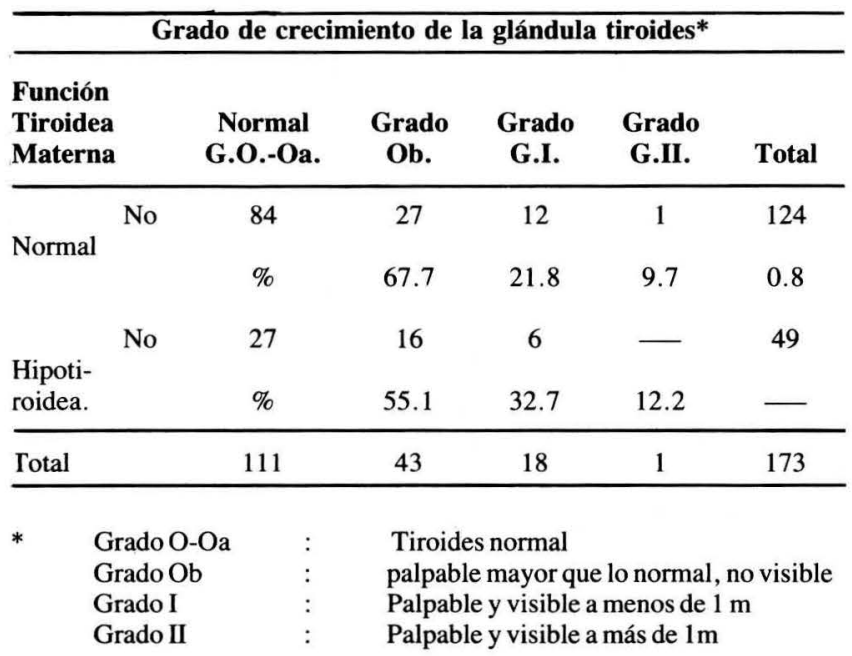

\section{Función tiroidea materna y duración del embarazo}

Se observó (cuadro 12) que 72 de los 78 hijos (92\%) de mujeres eutiroideas nacieron a término, mientras que el mismo hecho sólo se observó en 11 de los 15 (73\%) nacidos vivos de mujeres hipotiroideas. Entre los hijos nacidos antes del término, hubo una mayor proporción (dos de 15 vs. seis de 78) de hijos de madres hipotiroideas. Los dos únicos nacidos postérmino son hijos de madres de este mismo grupo.

\section{Función tiroidea materna y peso y talla de los recién nacidos}

No se encontró diferencia estadísticamente significativa al correlacionar el peso al nacer de los hijos de madres
Cuadro 12

FUNCION TIROIDEA MATERNA Y DURACION DEL EMBARAZO

\begin{tabular}{|c|c|c|c|c|c|}
\hline \multirow{2}{*}{$\begin{array}{l}\text { Función } \\
\text { Tiroidea } \\
\text { Materna }\end{array}$} & & \multicolumn{3}{|c|}{ Duración del embarazo* } & \multirow[b]{2}{*}{ Total } \\
\hline & & $\begin{array}{c}\text { Pretér- } \\
\text { mino }\end{array}$ & $\begin{array}{l}\text { A tér- } \\
\text { mino }\end{array}$ & $\begin{array}{c}\text { Postér- } \\
\text { mino }\end{array}$ & \\
\hline \multirow{3}{*}{ Normales } & No & 6 & 72 & - & 78 \\
\hline & $\%$ & 7.7 & 92.3 & - & \\
\hline & No & 2 & 11 & 2 & 15 \\
\hline \multicolumn{2}{|l|}{$\begin{array}{l}\text { Hipotiroi- } \\
\text { deas. }\end{array}$} & $\%$ & 13.3 & 73.3 & 13.3 \\
\hline \multicolumn{2}{|l|}{ Total } & 8 & 83 & 2 & 93 \\
\hline \multicolumn{2}{|c|}{$\begin{array}{l}\text { Pretérmino } \\
\text { A término } \\
\text { Postérmino }\end{array}$} & \multicolumn{3}{|c|}{$\begin{array}{ll}: & \text { de } 37 \text { semanas o menos } \\
: & \text { entre } 38 \text { y } 42 \text { semanas } \\
: & \text { de } 43 \text { o más semanas }\end{array}$} & \\
\hline
\end{tabular}

eutiroideas con aquellos de las hipotiroideas, aunque 53 de 78 hijos (68\%) de mujeres normales y 9 de $15(60 \%)$ neonatos de madres hipotiroideas nacieron con peso normal. La distribución para ambos grupos de R.N. con bajo peso y sobrepeso fue similar, con una mayor dispersión de los datos para el grupo de hijos de madres hipotiroideas (Cuadro 13).

$\mathrm{Al}$ correlacionar la talla de los recién nacidos con la F.T.M, llama la atención que hay una menor proporción de baja talla en los bebés de madres hipotiroideas que en los de mujeres normales y una mayor proporción de hijos con talla normal al nacer en el grupo de madres hipotiroideas, contrariamente a lo que podría esperarse. No hay diferencia estadísticamente significativa entre la talla de los hijos macrosómicos de madres normales y los de las hipotiroideas. (Cuadro 14).

\section{Cuadro 13}

FUNCION TIROIDEA MATERNA Y PESO NEONATAL

\begin{tabular}{|c|c|c|c|c|c|}
\hline \multicolumn{6}{|c|}{ Peso Neonatal* } \\
\hline $\begin{array}{l}\text { Función } \\
\text { Tiroidea } \\
\text { Materna }\end{array}$ & & $\begin{array}{l}\text { Baja } \\
\text { Peso }\end{array}$ & $\begin{array}{c}\text { Peso } \\
\text { Normal }\end{array}$ & $\begin{array}{l}\text { Sobre- } \\
\text { Peso }\end{array}$ & Total \\
\hline & No & 8 & 53 & 17 & 78 \\
\hline & $\begin{array}{c}\% \\
\text { No }\end{array}$ & $\begin{array}{c}10.3 \\
2\end{array}$ & $\begin{array}{c}67.9 \\
9\end{array}$ & $\begin{array}{c}21.8 \\
4\end{array}$ & 15 \\
\hline $\begin{array}{l}\text { Hipotiroi- } \\
\text { deas. }\end{array}$ & $\%$ & 13.3 & 60.0 & 26.7 & \\
\hline Total & & 10 & 62 & 21 & 93 \\
\hline $\begin{array}{l}\text { Bajo peso } \\
\text { Peso normal } \\
\text { Sobre-peso }\end{array}$ & $\begin{array}{ll}: & \text { de } \\
\vdots & \text { en } \\
\vdots & \text { de }\end{array}$ & $\begin{array}{l}500 \mathrm{go} \\
2.501 \mathrm{y} \\
601 \mathrm{go}\end{array}$ & $\begin{array}{l}\text { os } \\
00 \mathrm{~g}\end{array}$ & & \\
\hline
\end{tabular}

Al comparar el estado de F.T.M. con el sexo del hijo sorpresivamente encontramos que 10 de los 15 hijos (66.7\%) nacidos de mujeres hipotiroideas son de sexo femenino. 
Cuadro 14

FUNCION TIROIDEA MATERNA Y TALLA DEL RECIEN NACIDO

\begin{tabular}{|c|c|c|c|c|c|}
\hline \multicolumn{6}{|c|}{ Talla del recién nacido } \\
\hline $\begin{array}{l}\text { Función } \\
\text { Tiroidea } \\
\text { Materna }\end{array}$ & & $\begin{array}{l}\text { Baja } \\
\text { Talla }\end{array}$ & $\begin{array}{l}\text { Talla } \\
\text { Normal }\end{array}$ & $\begin{array}{l}\text { Macro- } \\
\text { sómico }\end{array}$ & Total \\
\hline \multirow{2}{*}{ Normales } & No & 9 & 64 & 5 & 78 \\
\hline & $\%$ & 11.5 & 82.1 & 6.4 & \\
\hline \multirow[b]{2}{*}{$\begin{array}{l}\text { Hipotiroi- } \\
\text { deas. }\end{array}$} & No & 1 & 13 & 1 & 15 \\
\hline & $\%$ & 6.7 & 86.7 & 6.7 & \\
\hline Total & & 10 & 77 & 6 & 93 \\
\hline
\end{tabular}

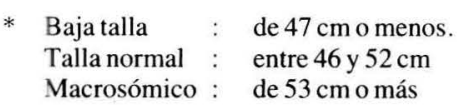

\section{Función tiroidea neonatal y otras variables}

Al comparar el grado de Función Tiroidea de los Hijos (F.T.H.) con estas mismas variables, en primer lugar, con el tamaño de la tiroides materna, se encontró que no hubo hijos con hipofunción tiroidea cuyas madres tuvieran un crecimiento de la tiroides que llegara a ser visible por el examinador; es decir, que en ningún caso sobrepaso el grado $\mathrm{Ob}$.

Excepto un caso de un hijo sub-normal que fue prematuro, todos los demás neonatos con función tiroidea anormal nacieron a término.

En todos los casos, los hijos con función tiroidea anormal tuvieron peso y talla al nacer dentro de límites normales.

No se encontró bocio en ninguno de los recién nacidos examinados.

En el grupo de siete "Embarazos anormales" se encontró que tres madres eran hipotiroideas y entre las tres dieron origen a una mola hidatiforme, un aborto y un mortinato. En las cuatro mujeres eutiroideas restantes se presentaron dos recién nacidos vivos que murieron antes del quinto día de edad, un aborto y un mortinato.

\section{Conclusiones y comentarios}

Es sorprendente que en Colombia, área de bocio endémico, no se encuentren trabajos de investigación que relacionen las funciones tiroideas de la madre y su hijo. Hasta donde conocemos, éste es el primer trabajo colombiano en el que se busca determinar la influencia del hipotiroidismo materno sobre la función tiroidea de su descendencia. Esperamos que los resultados de este informe contribuyan a estimular el desarrollo de investigaciones más extensas y profundas sobre este fascinante tema.

Por haberse realizado en un solo hospital con un área de influencia limitada, a pesar de su amplia cobertura, no se intenta extrapolar las conclusiones a otra población diferente de la que asiste a la consulta de control prenatal del H.M.I. de Bogotá.

En efecto, el $96 \%$ de la población estudiada es procedente de Bogotá D.E., y como era de esperarse, por tratarse de mujeres en edad reproductiva, el $60 \%$ tiene entre 21 y 30 años.

El hecho de encontrar el $32.3 \%$ de las gestantes eutiroideas con algún grado de crecimiento anormal de la tiroides (bocio) está de acuerdo con descripciones previas que refieren un aumento del tamaño de la tiroides durante el embarazo normal $(3-7,29)$, aunque hay otros reportes que indican que esto sólo ocurre en áreas de bocio endémico, principalmente por baja ingesta de yodo en la dieta $(1,6,32,33)$. Al mismo tiempo llama la atención, y no tenemos otra explicación que la carencia de un segundo o tercer examinador, que el 55\% (27 de 49) de las embarazadas hipotiroideas no tuvieran bocio.

No se encontraron diferencias estadísticamente significativas entre la duración del embarazo, peso, talla ni sexo de los hijos de mujeres eutiroideas y los de las hipotiroideas, pero llaman la atención dos hallazgos:

1. Hubo una menor proporción de baja talla en los R.N. de mujeres hipotiroideas que en los hijos de mujeres normales, lo cual es contrario a lo encontrado en modelos experimentales en animales en los cuales los hijos de ratas tiroidectomizadas tenían un menor peso que los hijos de ratas normales $(10,11,13-16)$. Esta aparente discrepancia puede tener origen en el hecho de que las ratas madres eran severamente hipotiroideas (tiroidectomizadas) en tanto que en el presente estudio se trata de mujeres con hipotiroidismo subclínico.

2 . Se encontró una mayor proporción (2/3) de sexo femenino en hijos de mujeres hipotiroideas, y dos de los tres R.N. hipotiroideos congénitos son niñas. Aunque el reducido número de casos no le otorgue significación estadística a estos datos, ellos son coincidentes con la reconocida observación de que los problemas tiroideos son más frecuentes en mujeres que en hombres (1). Por otra parte, de repetirse estos hallazgos en estudios de mayor alcance (mayor proporción de hijas con hipotiroidismo) se estaría autorizado a pensar que éste es un factor que tiende a perpetuar la disfunción en zonas de bocio endémico, pues estas mujeres hipotiroideas al reproducirse, no sólo prolongan sino que magnifican el problema.

Uno de los hallazgos a destacar en el presente informe es la alta proporción de hipotiroidismo materno encontrada: 49 de 173 mujeres $(28.3 \%)$ cuyas determinaciones de TSH post-TRH excedieron el límite aceptado como normal.

De estos 49 casos solamente tres tuvieron la TSH basal anormalmente elevada. De haberse tomado esta última prueba (de igual especificidad pero de mucha menor sensibilidad que la de la TSH post-TRH) como base exclusiva para el diagnóstico, hubiéramos dejado de reconocer el $49 \%$ de las mujeres hipotiroideas. De todos modos, el $28 \%$ de hipotiroidismo materno encontrado en este trabajo, nos parece una proporción muy elevada ya que esto significa que uno de cada 3.5 gestantes (aproximadamente tres de cada 10) que consultan al H.M.I. son hipotiroideas en fase reproductiva.

Más importante aún, nos parece la tasa de hipofunción tiroidea encontrada en los R.N. Siete de los 123 neonatos (5.7\%) tuvo algún grado de hipofunción tiroidea (cuatro (3.3\%) con función tiroidea subnormal y tres $(2.3 \%)$ hipotiroideos congénitos), cifras similares a las encontradas en estudios de detección de hipotiroidismo congénito realizados 
en el H.M.I. de Bogotá (35) y en Ecuador (28) (Aproximadamente $2 \%$ ). Estos informes, incluido el presente, difieren enormemente de los reportados en países que no son áreas de bocio endémico en donde la proporción de hipotiroidismo congénito oscila entre 1: 4.000-5.000 nacidos vivos (0.02\%) $(1,2,4-7,31)$.

Teniendo en cuenta solamente las 93 parejas madre-hijo del cuadro 10 , hay tres casos $(3.2 \%)$ en las cuales ambos miembros tienen hipofunción tiroidea, y en dos de esos" casos $(2.1 \%)$ los hijos son hipotiroideos congénitos. Además hay dos parejas en las cuales la madre es eutiroidea y los hijos son, uno subnormal y otro hipotiroideo congénito, resultando entonces una proporción global de hipofunción tiroidea neonatal del $5.3 \%$ en este grupo de 93 parejas.

Sabiendo las consecuencias que la hipofunción tiroidea fetal y neonatal tienen sobre el desarrollo particularmente cerebral del individuo $(1-3,10,11,27,30)$, podemos tener una idea de la magnitud del problema aquí presentado.

Como ha sido postulado por otros autores, creemos que no existe actualmente razón científica para permitir que siga presentándose lesión cerebral irreversible causada por hipotiroidismo congénito en hijos de madres hipotiroxinémicas debido a la baja ingesta de yodo en las áreas de bocio endémico $(10,11)$; ya que la hipofunción tiroidea neonatal es una de las pocas alteraciones congénitas endocrinas en las que resulta muy barato, sencillo y efectivo el tratamiento farmacológico de suplencia hormonal, el cual entre más temprano se inicie previene en forma más efectiva las consecuencias que este tiene sobre el desarrollo físico y principalmente neurológico e intelectual de los afectados (36); esto adquiere más significación en áreas donde el bocio endémico es mayor $(1,28)$. Es posible que las consecuencias del hipotiroidismo materno y neonatal, junto con la desnutrición, jueguen un papel en las hondas diferencias que nos separan de los países desarrollados (28).

Consideremos que un método de tamizaje, basado en la cuantificación sérica de la TSH-NN $(35,36)$ para determinar su grado de función tiroidea estaría ampliamente justificado aún si con ello se lograra solamente el descubrimiento de unos pocos casos de hipofunción tiroidea neonatal, lo que permitiría la aplicación oportuna de medidas terapéuticas sencillas y ampliamente efectivas $(3,-5,9,19)$. En este mismo sentido, la búsqueda de hipofunción y las consecuentes medidas terapéuticas por parte del médico para asegurar el mantenimiento de una función tiroidea normal en toda mujer embarazada sería una medida preventiva para hipotiroidismo congénito y sus secuelas como el subdesarrollo mental de la descendencia en zonas de bocio endémico $(9,10,19,21$, 36).

Este es un estudio transversal que observa un fenómeno de relativa "baja frecuencia" y que como los de su tipo, necesita una muestra mayor para que el fenómeno investigado aparezca. Los resultados aquí presentados, obtenidos sobre una población relativamente pequeña sugieren que la F.T.M. tiene una relación directa con la neonatal. Para confirmar este concepto, es de la mayor conveniencia la ejecución de estudios epidemiológicos orientados a verificar esta hipótesis en grupos poblacionales y en otras regiones dl país. De encontrarse proporciones de hipotiroidismo materno y fetal y relaciones entre las F.T.M. y neonatal similares a las informadas por nosotros sobre muestras representativas de sectores de población a nivel seccional o nacional, se habrá dado un paso fundamental en el mejoramiento de la salud y para el progreso del pueblo colombiano.

\section{Agradecimientos}

Al Doctor Bernardo Reyes Leal por sus valiosas enseñanzas y despertar nuestro interés en este tema.

Al Doctor Enrique Guerrero Medina por su constante apoyo y colaboración en el desarrollo de este trabajo.

Al Ingeniero Edgar Rodríguez por sus aportes en el manejo de estadística de los datos de este informe.

A la Doctora Reina E. de Alayón por su participación en la obtención de los datos de laboratorio.

A todas las madres que junto con sus hijos hicieron posible el desarrollo de este estudio.

$\mathrm{Y}$ a todas las personas que de una $\mathrm{u}$ otra manera colaboraron en el desarrollo de la investigación y elaboración del informe.

\section{BIBLIOGRAFIA}

1. Camargo C. Hypothyroidism and goiter during pregnancy. In: Brody $\mathrm{S}$, Ueland $\mathrm{K}$, eds; Endocrine disorders in pregnancy. First Edition. Appleton and Lange: 1989; 165-176.

2. Taylor S., Williams T. The thyroid and parathyroid glands in pregnancy. In: Shearman R, eds; Clinical Reproductive Endocrinology. First Edition. Churchill Livingstone: 1985; 327-337.

3. Ingbar S. The thyroid gland. In: Wilson J, Foster D. eds. Williams Textbook of Endocrinology. Seventh Edition. Philadelphia (Pa): W. B. Saunders, Rev. Ed. Of: Textbook of Endocrinology. First Edition: $1981 ; 682-815$.

4. Mestman G. Enfermedades tiroideas durante el embarazo. Clin. Perinatol 1985; 3: 679-696.

5. Mestman G. Asistencia de las enfermedades tiroideas durante el embarazo. Clin. Perinatol; 1980; 2: 383-398.

6. Burr W. Thyroid disease. Clinics in Obstetrics and Gynecology; 1986; 13(2): 277-290.
7. Innerfield R., Hollander C. Thyroidal complications of pregnancy. Medical Clinics of N.A.; 1977; 61(1): 67-86.

8. Montoro M., Collea J., Frasier D. et al. Successful outcome of pregnancy in women with hypothyroidism. Annals of Internal Medicine 1981; 94: 31-34.

9. Carr E., Beierwaltes W., Raman G. et al. The effect of maternal thyroid function of fetal thyroid function and development. J.C.E.M.; 1959; 19(1): 1-18.

10. Morreale de Escobar G. Thyroid hormones and the developing brain. In: Madeiros-Neto, Gaitan, eds; Frontiers of thyroidology. Plenum Medical: 1986; 5-17.

11. Morreale de Escobar G., Obregon MJ., Escobar del Rey F. Fetal and maternal thyroid hormones. Hormone Res; 1987; 26: 12-27.

12. Obregon MJ., Mallol J., Pastor R. et al. L-Thyroxine and 3, 5, 3 triiodo-1-thyronine in rats embryos before onset of fetal thyroid function. Endocrinology; 1984; 114(1): 305-307. 
13. Morreale de Escobar G., Pastor R., Obregon MJ. et al. Effects of maternal thyroidectomy on rat embryonic T4 and T3 contents and development, before and after onset of fetal thyroid function. In: Madeiros-Neto, Gaitan, eds; frontiers of thyroidology. Plenum Medical: 1986; 1177-1181.

14. Escobar del Rey F., Pastor R, Mallol J. et al. Effects of maternal iodine deficiency on T4 and T3 contents of rat concepta, both before and after onset of fetal thyroid function. In: Madeiros-Neto, Gaitan, eds; frontiers of thyroidology. Plenum Medical: 1986; 1033-1038.

15. Morreale de Escobar G., Pastor R, Obregon MJ. et al. Effects of maternal hypotiroidism on the weight and thyroid hormone content of rat embryonic tissues, before and after onset of fetal thyroid function. Endocrinology 1985; 117(5): 1890-1900.

16. Morreale de Escobar G., Obregon MJ., Ruiz de Oña C. et al. Comparison of maternal to fetal transfer of 3, 5, 3 triiodothyronine versus thyroxine in rats, as assessed from 3, 5, 3 triiodothyronine levels in fetal tissues. Acta Endocrinologica 1989; 120(1): 20-30.

17. Raiti S., Holzman G., Scott R. et al. Evidence for the placental transfer of triiodothyronine in human beings. New England Journal of Medicine 1967; 277(9): 156-159.

18. Vulsma T., Gons M., Vijlder J. Maternal fetal transfer of thyroxine in congenital hypothyroidis due to a total organification defect or thyroid agenesis. New England Journal of Medicine 1989; 321(1): 13-16.

19. Larsen P. Maternal thyroxine and congenital hypothyroidism. New England Journal of Medicine 1989; 321(1): 44-46.

20. Fisher D., Klein A. Thyroid development and disorders of thyroid function in the newborn. New England Journal of Medicine 1981; 304(12): 702-712.

21. Man E., Serunian S. Thyroid function in human pregnancy IX. Development or retardation of 7-years old progeny of hypothyroxinemic women. Am. J. Obstet. Gynecol. 1976; 125(7): 949-957.

22. Silva E., Leonard J. Regulation of rat cerebrocortical and adenohypophyseal type II 5-Deiodinase by Thyroxine, Triiodothyronine, and reverse Triiodothyronine. Endocrinology 1985; 116(4): 1627-1635.

23. Bernal J., Pekonen F. Ontogenesis of the nuclear 3, 5, 3-Triiodothyronine receptor in the human fetal brain. Endocrinology 1984; 114(2): 677-679.
24. Ferreiro B., Bernal J., Goodyer C. et al. Estimation of nuclear thyroid hormone receptor saturation in human fetal brain and lung during early gestation. J.C.E.M. 1988; 67(4): 853-856.

25. Lastra G. Función tiroidea en bocio endémico. IV. Encuentro de egresados facultad de medicina Universidad Nacional. AEXMUN. Congreso de especialidades clínicas y ciencias básicas afines. 1989.

26. Santisteban P., Obregon MJ., Rodríguez A. et al. Are iodine-deficient rats euthyroid? Endocrinology 1982; 110: 1780-1789.

27. Thilly C., Delange F., Lagasse R. et al. Fetal hypothyroidism and maternal thyroid status in severe endemic goiter. J.C.E.M. 1978; 47(2): 354-360.

28. Varea J., Paredes M., Ramírez I. et al. Detección de hipotiroidismo congénito. In: Varea J. Paredes M. Ochoa C, eds; Fundación ciencia para estudio del hombre y la naturaleza. Quito, Ecuador 1987; 156-202.

29. Kannan V., Sinha M., Devi P. et al. Plasma thyrotropin and its response to thyrotropin releasing hormone in normal pregnancy. Obstetrics and Gynecology 1973; 42(4): 547-549.

30. Evered DC., Ormonston BJ., Smith PA. et al. Grades of hypothyroidism Br. Med. J. 1973; 1: 657-662.

31. Alm J., Hagenfeldt L. et al. Incidence of congenital hypothyroidism: retrospective study of neonatal laboratory screening vs. clinical symptoms as indicators leading to diagnosis Br. Med. J. 1984; 289 : 1171-1175.

32. Levi R. et al. The myth of goiter in pregnancy. Am. J. Obstet Gynecol 1980; 137(6): 701-703.

33. Crooks J., Tulloch M., Turnbull AC. et al. Comparative incidence of goiter in pregnancy in Ireland and Scotland. Lancet 1967; 2: 625.

34. Patel Y., Pharoah P., Hornabrook R. et al. Serum triiodothyronine, thiroxine and thyroid stimulating hormone in endemic goiter: a comparison of goitrous and nongoitrous subjects in New Guinea. J.C.E.M. 1973; 37: 783 .

35. Carrillo J., Carrascal R., de Alayón R. Detección de hipotiroidismo congénito mediante la dosificación de T4 y TSH neonatales. Actas primer curso bianual. Departamento de Medicina Interna Universidad Nacional de Colombia: 1982; 176-184.

36. Klein A., Foley T., Larsen R. Neonatal Thyroid function in congenital hypothyroidism. Journal of Pediatrics 1976; 89(4): 545-549. 